\section{0 second working time and 10 second cure}

Radical amplified photopolymerisation (RAP) is one of several breakthroughs that won the Dental Advisor's Best Universal Composite Award nine years in a row for Tokuyama's Estelite.

The essential feature of the RAP technology is its ability to photopolymerise in only a third of the time required for conventional composites based on camphorquinone and amine initiation.

The composite is very stable under ambient light giving at least 90 seconds of working time. However, under the curing light, RAP initiates multiple energised monomer molecules which gives both a rapid and more complete cure.

Normally these two factors, working time and curing time, work against each other. Fast curing leads to a short working time. Long working time leads to a long curing time. RAP technology overcomes this problem, giving the best of both worlds.

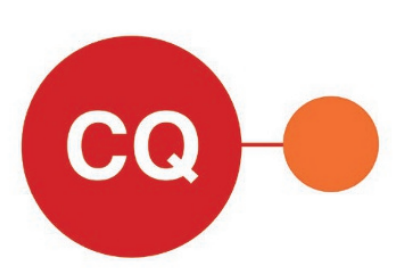

Fig. 1 Traditional composites
In traditional composites, each CQ molecule energises one monomer molecule per cycle (Fig. 1). In Estelite RAP technology, multiple monomer molecules are energised per cycle (Fig. 2), so less CQ is required.

With the composite more stable in ambient light, the dentist has time to utilise the full aesthetic capabilities of Estelite to create beautiful restorations that stay looking beautiful. With less $C Q$, there is minimal colour change after curing, further helping to achieve this perfection, says the manufacturer.

Light energises the CQ which is then transferred to the monomer. Having lost its energy, the CQ is once more energised by the light and so on, until curing is achieved.

Together with other breakthroughs such a spherical filler pearls, the Estelite Advantages are:

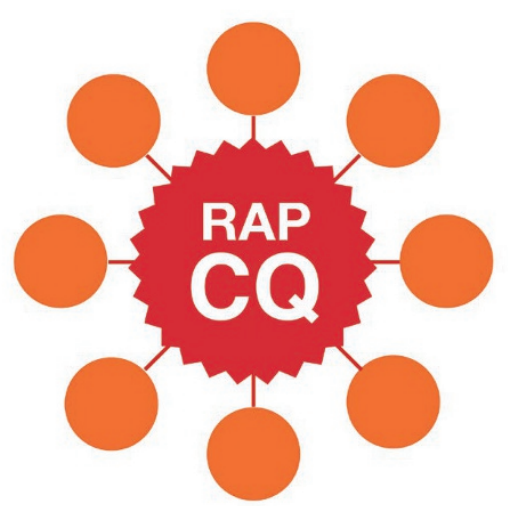

Fig. 2 Estelite RAP technology
- Extended

working time - at least 90 seconds

- Quick curing time - 10 seconds

- Exceptional handling

- Superb fast, long-lasting polish

- Superior shade matching

- High wear resistance and little abrasion to opposing teeth

- Minimal shrinkage after light curing (1.3\% linear).

For more information, call 01274885544 or visit www.trycare.co.uk/estelite.

\section{The difference between using a device or investing in a philosophy}

AquaCare uses breakthrough technology to deliver a comfortable, quick, clean procedure, by using a fine stream of fluid combined with a tiny volume of powder directed at the teeth to be treated.

Unlike conventional rotary cutting instruments, the AquaCare handpiece is not in direct contact with the tooth structure, removing only the minimum of sound tooth material and eliminating the risk of chipping and stress fractures.

The company has designed AquaCare so that it works ideally and provides for the widest range of dental cases.

Its unique cartridge system allows for two chambers to be ready for use at any time; multiplying your options to adapt to any case or situation. It takes just one second to switch. As more powders become available the system is future proof.

Its capabilities include adhesive dentistry, bonding, cleaning, desensitising, endo, hygiene, implant, laboratory, MI, ortho, paediatric, Pekkton, perio, remineralising, restorative dentistry, stain removal, veneers, supra or sub gingivally.

More information is available by visiting https://velopex. com/community/ or https://www.instagram.com/velopexuk/ or by emailing aquacare@velopex.com.

\section{Al software traces a perfect ID nerve in 4.5 seconds - a first in dental history}

CT Dent is at the forefront of technological advances such as the use of artificial intelligence (AI) in dentistry which is dedicated to teaching computers how to automate radiological diagnostics using deep learning.

Its use is increasing, and CT Dent says its AI software recently traced a perfect ID nerve in 4.5 seconds - the first time in dental history.

Machine learning provides a point of view that was not previously possible through human diagnosis. It is a technique in computer sciences to find and train algorithms to solve tasks using real examples, rather than explaining exactly how to do it.

In addition to using AI for ceph tracing reports, CT Dent has spent a considerable amount of time developing and testing its own machine learning artificial intelligence.

It allows the company to process scans in a matter of seconds with a $99.7 \%$ success rate. Although accurate, for additional quality control, the company's processing team still check the scan whilst the evolution of this AI continues to gain pace.

For further information on services or to register a practice, please visit www. ct-dent.co.uk or call 02074875717 . 